\title{
TDRHN: A Threshold Sensitive Dynamic Responsive Hybrid Network Protocol based on CTP
}

\author{
Xingming Sun ${ }^{1,2,3}$, Chengju Xue ${ }^{1}$ and Baowei Wang ${ }^{1,2,3}$ \\ ${ }^{1}$ School of Computer and Software, Nanjing University of Information Science \& \\ Technology, Nanjing 210044, China \\ ${ }^{2}$ Jiangsu Engineering Center of Network Monitoring, Nanjing 210044, China \\ ${ }^{3}$ Jiangsu Collaborative Innovation Center on Atmospheric Environment and \\ Equipment Technology, Nanjing 210044, China \\ sunnudt@163.com,xcj2550@163.com,wbw.first@163.com
}

\begin{abstract}
The protocols of Wireless Sensor Networks (WSNs) can be categorized into two classes: proactive network protocol like CTP and reactive network protocol like TEEN. The former periodically collects data, working well in the applications of collecting data persistently. However, it can't dynamically alter the transmission frequency and the routing selecting strategy. The latter can react to the environment change, so it is widely used in the applications requiring nodes to be reactive to some emergencies. But it only works in reactive networks without collecting data under normal circumstance. We find that dynamically altering the transmission frequency and the routing selecting strategy according to the environment change is very effective and significant. For the reasons above, this paper presents TDRHN protocol. It periodically collects data in normal situation and can not only take faster transmission frequency but also alter the routing selecting strategy when it detects some emergencies. So TDRHN can effectively work in hybrid networks combining proactive networks and reactive networks. Finally, we evaluate TDRHN on the testbed with Telos B motes in real deployment. The concluded results of the experiments demonstrate that TDRHN has some particular advantages.
\end{abstract}

Keywords: WSN; TDRHN; transmission frequency; routing selecting strategy; emergency

\section{Introduction}

Owning to the rapid development of sensor technology and embedded computing, the WSN has been widely utilized in many applications such as environmental monitoring, alarming system, military security, and so on [1-3]. A WSN is composed of many sensors that have limited computing ability and energy. Comparing with traditional technology, it is more low-cost and not limited in space as special terrain. Thereby, many researchers are dedicated to the development of WSNs. To solve the communication and collecting data problem, many sorts of protocols and algorithms are proposed. Such as Location-Aware Routing Protocol (LARP) [11] for UWSNs uses the location information of nodes to promote the transmission reliability. ADCMCST [12] is an approximation algorithm to construct a tree network for homogeneous wireless sensor network, it can balance the payload of each node and consequently prolong the network lifetime. They are divided into two categories based on whether they can support the response to environment change: proactive protocol like CTP [4] and reactive protocol like TEEN [5]. CTP is a reliable plane routing protocol, which routing selecting strategy is based on ETX (Expected Transmissions Value), and the strategy does not take residual energy into account. TEEN is a reactive clustering protocol, it only collects data when the nodes 
detect the emergency. However, proactive network protocol periodically collects data in normal situation without supporting the response to environment change. On the contrary, reactive network protocol only reacts to environment change without collecting data in normal situation.

A protocol to be widely used in many environments should not only collect data under normal circumstance but also can react to emergencies. The environment in which sensor nodes are deployed tends to be unpredictable, sometimes it may encounter some emergencies such as forest fires, in this case, terminal monitoring staffs hope to know what is happening in the monitoring area as soon as possible. So nodes that have sensed the emergency should take faster frequency of sending data to sink station. Faster transmission leads to consuming more energy in short time, so the residual energy in nodes should be considered in the routing selecting mechanism. To adapt and react to the environment, the transmission frequency and the routing selecting strategy in normal situation are different from that in burst situation.

TDRHN, this paper presented, is based on CTP that provides reliable routing mechanism, combining CTP's proactive mechanism and TEEN's reactive mechanism. It periodically collects data in normal situation, in the meantime, it can react to the burst event in the way that it takes faster transmission frequency of the node monitoring the event and adjusts routing just including the transmission path of the node. Finally the rate of sending data and changed routing selection strategy will return to the original state when the node detecting the emergency returns to normal state. By doing so, we can implement a protocol that effectively works in the normal environment and can react to the emergency.

TDRHN organizes the global network and collects data at the beginning like CTP as shown in Figure.1, and it will keep this state as long as there is no node monitoring the emergency. But when the emergency appears, TDRHN will make some changes. As we can see from the Figure.2, when Node 10 detects the emergency, TDRHN will take two actions to react to the situation. The first action is that Node 10 will immediately take faster frequency of sending data to its next hop node. The second action is that Node 10 selects its parent node on the base of new metric combining ETX with Energy. Node 10 selects Node 7 as its parent node rather than Node 6, then Node 7 sends data to Node 4, and Node 4 transmits data to Node 1 . That is, the routing selecting strategy of the path from Node 10 to the sink has changed, while other routes that do not include the path keep before. When the emergency disappears, Node 10 will send data in the rate of that in normal, and the routing selecting strategy will return to the original state.

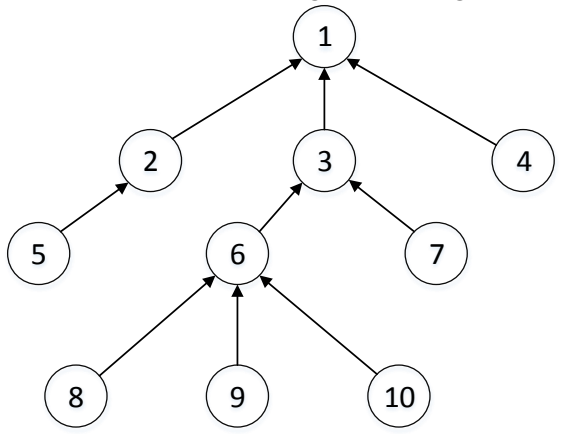

Figure 1. The Topology in Normal Situation

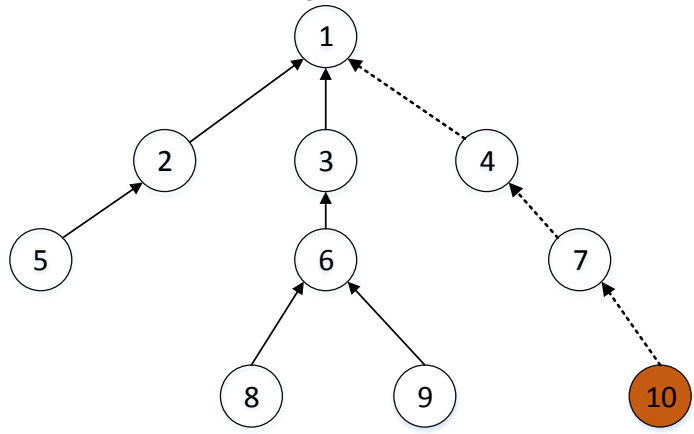

Figure 2. The Topology when Node 10 Detects the Emergency

This paper's contributions can be concluded as follows. First, we implement a protocol that can not only collect data in normal situation but can react to the burst situation. Secondly, the transmission frequency of a node is dynamically adjusted according to the environment change. Thirdly, the routing selection strategy can dynamically change in the term of the environment change. 
This paper is organized as follows. In section 2, we give the related works about proactive and reactive protocols in WSNs. In section 3, we introduce the details of our presented protocol. In section 4, we test TDRHN in real experiments, and evaluate the results. In section 5, the conclusions of the study and the future work are given.

\section{Related work}

Currently the protocols are divided into two types: Proactive Network Protocol and Reactive Network Protocol. The former is presented to serve for proactive networks, such as CTP, which periodically collects data. The latter can support the response to emergencies in a reactive network. However, in the real applications, technical people tend to employ hybrid networks to meet various demands.

\subsection{Proactive Network Protocol}

Proactive network protocol is designed to periodically monitor the environment or targets. Its features are demonstrated as bellow:

All nodes sense the data from the environment and periodically send data to the sink.

Even if the node encounters the emergency, it still keeps original state without changing the period of sending data and the routing selection strategy.

CTP (Collection Tree Protocol) is one of these protocols, it is a tree-based protocol. One node in the network is set to root, other nodes form the routing paths to the sink on the base of ETX. CTP is an address-free algorithm in the sense that a node does not select a fixed root as its destination; instead, by selecting a next node as its transmission station, it forms a route to the sink implicitly. That is, CTP takes advantage of the multi-hop pattern to transmit packets from sensor nodes to the sink. In accordance with routing gradient, nodes form the transmission routes.

CTP is a reliable and robust data collection protocol, which is independent of hardware. Studies show that its delivery rate reaches 90 percent [4]. It can effectively refrain repeat packets and routing loops problems by using ACK (Acknowledge Check) and THL (Time Has Lived).

However, it also has some drawbacks .One is that it can't meet the need of reactive networks, because it can't react to the change in the surrounding environment. Second, its routing selecting does not take energy into consideration, leading to some nodes transmitting large quantities of packets to dead prematurely.

\subsection{Reactive Network Protocol}

TEEN (Threshold sensitive Energy Efficient sensor Network protocol) is the first protocol developed for reactive networks, and it is a cluster-based protocol on the base of LEACH [6].

TEEN implements the response to the emergency by setting Hard Threshold and Soft Threshold. The node will transmit data to its next cluster head, only when both following conditions are true.

1. The current value of the sensed attribute is greater than the hard threshold.

2. The current value of the sensed attribute differs from SV (the sensed value, which is stored in an internal variable in the node) by an amount equal to or greater than the soft threshold.

Whenever a node transmits data, SV is set equal to the current value of the sensed attribute.

Thus, the hard threshold and the soft threshold reduce the number of transmissions by allowing the nodes to transmit only when the sensed attribute is in the range of interest, so the nodes in the global network can run more time. In addition, the hard threshold and the soft threshold can be set repeatedly to meet many sorts of applications. 
The main drawback of this scheme is that, if the thresholds are not reached, the nodes will never send data, so the user will not get any data from the network at all and will not come to know even if all the nodes die. Thus, this scheme is not well suited for applications where the user needs to get data on a regular basis. Moreover, its routing selecting strategy can't dynamically adapt to the environment change.

\subsection{Dynamic Transmission Frequency}

The optimal sense rate of the node in WSNs is studied by some researchers. Wendong Xiao and Lihua Xie [7] propose an adaptive sense rate in using target tracking. The scheme extends the Kalman filtering to track the target in lining moving, and the sense rate is dynamic adaptive. But the mechanism is not suitable for the applications that demand periodically collecting data, it does not support the response to emergencies. Currently, few researchers pay attention adjusting dynamically the node's transmitting frequency to meet the need of proactive networks and reactive networks.

\subsection{Dynamic Route}

The fixed route may no longer be suited for the changed environment or the residual energy of nodes, at this time, we hope the route dynamically changes. In [8], the authors propose a dynamic route theory, which can dynamically adjust the route by sensing the residual energy of nodes and the energy to be consumed in transmitting data to the next hop node. By doing so, the network prolongs its life span. Based on CTP, E-CTP [9] takes the residual energy into consideration in selecting the route. These protocols above mentioned, although their routes dynamically change, they do not react to emergencies. In addition, the metrics do not change to adapt to environment change.

\section{Design of TDRHN}

In this section, we present a hybrid network protocol called TDRHN (a Threshold sensitive Dynamic Responsive Hybrid Network protocol based on CTP). It is proposed to be used in the hybrid network that combines proactive networks and reactive networks, it can periodically collects sensed data to terminal in normal situation and support the response to burst situation.

\subsection{Design Goals and Challenges}

The target of HDRHN is not only to periodically transmit data in normal situation but to make some adjustments including transmission frequency and routing selection strategy when the nodes monitor some emergencies. The challenges to achieve the goal are summarized as follows.

First, TDRHN can collect data in normal situation and react to the emergency in the environment, so it is well suited for hybrid networks.

Second, the node keeps low frequency of transmitting data in normal, it will take high frequency to send data as monitoring the emergency, by doing so, TDRHN can dynamically adjust the rate of transmitting data in the term of the node's state.

Third, TDRHN forms the routing on the basic of ETX in normal situation, it will employ new metric taking advantages of ETX and Energy to adapt to the changed environment. So it can dynamically adjust the routing selecting strategy.

Proved in a number of real experiments in our laboratory, CTP has demonstrated that it is a reliable protocol. Based on RNP calculation [10], CTP's routing selecting metric called ETX reacts the situation of the communication link quality, thereby CTP's communication is stable and guaranteed. However, the metric does not considerate residual energy, leading to premature death of the nodes transmitting a large of packets. Hence, TDRHN is designed on the basic of CTP, it guarantees the communication quality 
and considers the residual energy element in burst situation.

\subsection{Overall Architecture}

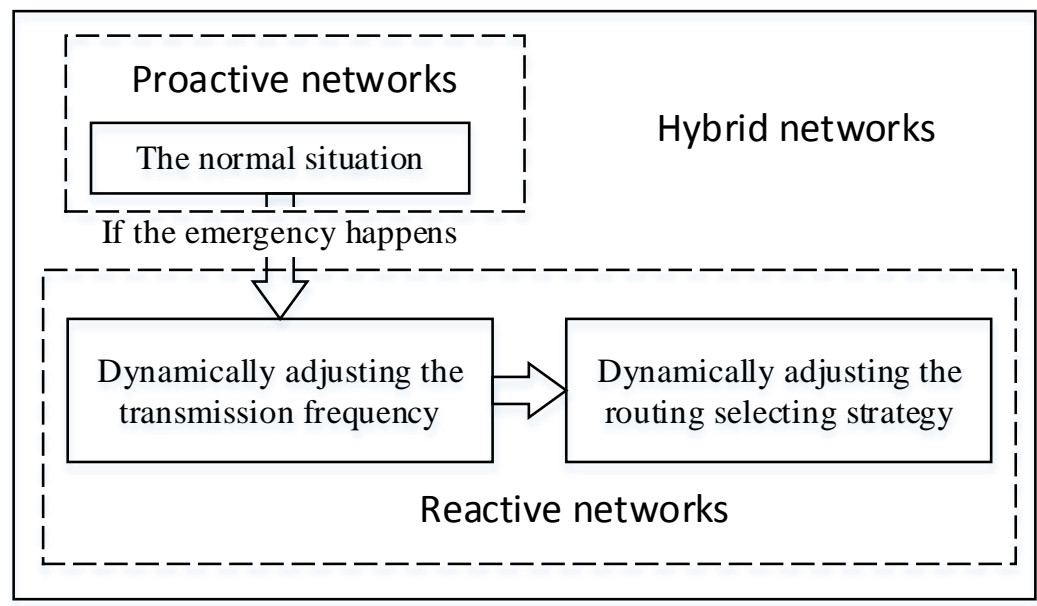

Figure 3. Frame Structure of TDRHN

TDRHN services for hybrid networks (as seen from Figure.3). All the nodes are firstly deployed in the normal situation. When a node monitors the emergency, that is, it is currently in burst situation, the node will dynamically adjust the transmission rate and the routing selecting strategy.

\subsection{Hybrid Network Protocol}

To combine proactive network protocol with reactive network protocol, TDRHN employs the threshold strategy.

Absolute Threshold (AT): This set threshold is the absolute value of the sensed attribute. This value can be set again to adapt different applications.

Relative Threshold (RT): This set threshold is equal to the absolute value of that current sensed value minus previous sensed value. This value can also be defined repeatedly according to various degrees.

The nodes will keep continuous monitoring the surrounding. The absolute threshold and the relative threshold are defined before the nodes begin to run, after that, these nodes can run with the set values. The sensed value is stored by embedding a variable in the node, called Flash Value (FV). TDRHN sets conditions to determine whether the node takes actions to the emergency. The conditions include two points.

First, the new sensed value is not less than absolute threshold.

Second, the current value of sensed attribute (called CV) differs from FV by an amount not less than relative threshold.

What is different from TEEN is that the nodes can periodically transmit data rather than transmitting data no longer when the conditions are not true. Moreover, in TDRHN, the nodes monitoring the emergency will increase the transmission frequency and change the routing selecting strategy. Hence, TDRHN can run effectively in hybrid network. 


\subsection{The Dynamic Transmission Frequency}

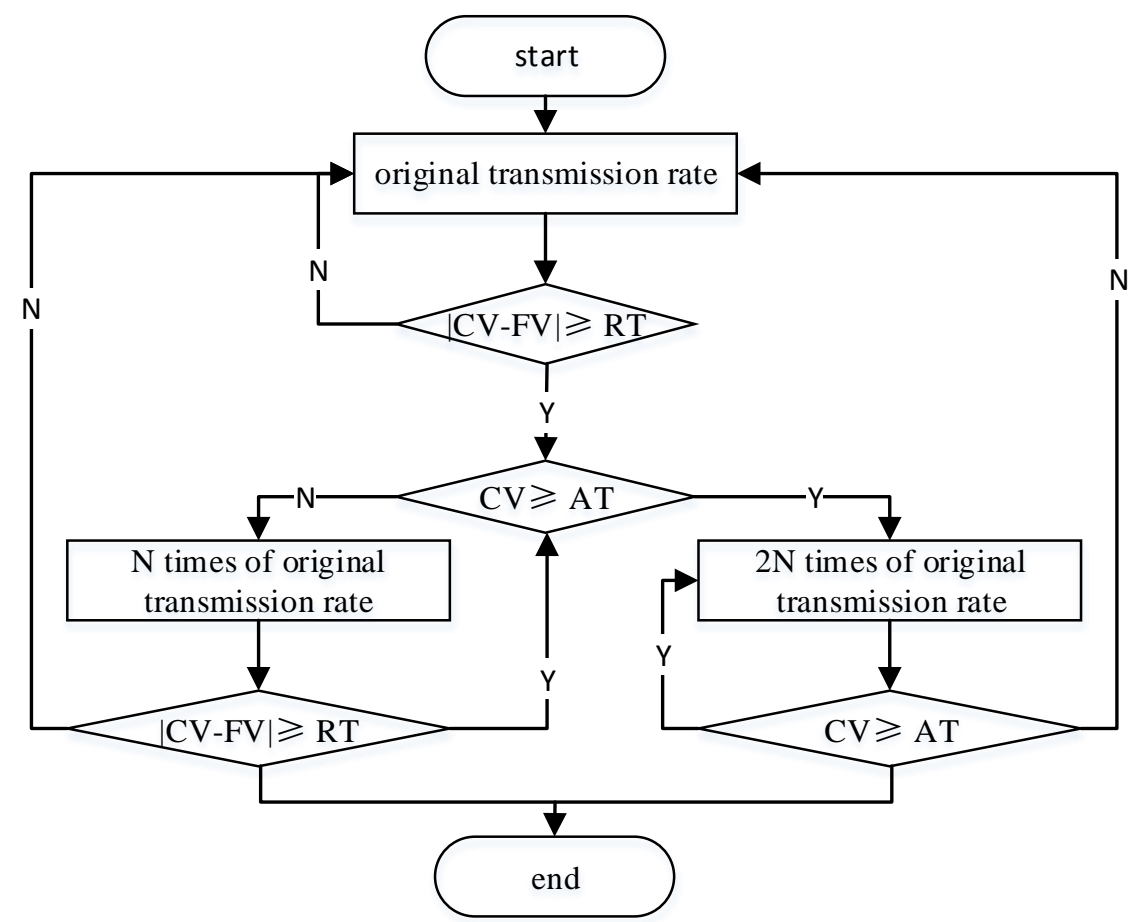

Figure 4. The Flow Chart of Transmission Frequency

From the Figure.4, we can clearly see the process of dynamically adjusting the transmission rate.

Step-1: At the beginning, the node is in proactive networks, it will periodically transmitting sensed data.

Step-2: If $|\mathrm{CV}-\mathrm{FV}| \geq \mathrm{RT}$, which means the node monitors the emergency, it will take faster rate to transmit data to the next hop node. Then if the condition that $C V \geq A T$ is satisfied, the transmission rate of the node will becomes $2 \mathrm{~N}$ ( $\mathrm{N}$ can be set in different applications) times of the original, after this, the node will keep this fast transmission situation as long as the condition that $\mathrm{CV} \geq \mathrm{AT}$ keeps true; when the condition is not satisfied, the node will back to Step-1, at this time, the node returns to the normal state and employs original transmission frequency. When the node judges that $|\mathrm{CV}-\mathrm{FV}| \geq \mathrm{RT}$ and $\mathrm{CV}<\mathrm{AT}$, the rate will be $\mathrm{N}$ times of the original, after that, the node will judge the condition that converts its original rate to $\mathrm{N}$ times of original rate.

\subsection{The Dynamic Routing Selecting Strategy}

Another thing to be done when the node monitors the emergency is that it will change the routing selecting strategy. Here, a new routing selecting metric, called EE (ETX Energy), is defined below.

$E E=E T X^{\alpha} E^{\beta}$

$\mathrm{E}$ is the node's residual energy, the voltage value stands for it here. $\alpha$ and $\beta$ are weighting factors that balance ETX and residual energy. Note that $\alpha$ is nonnegative, while $\beta$ is negative.

When the node takes higher frequency of transmitting data, the route including the node detecting the emergency will form a new route on the basis of EE. The node will select the sensor node that has the least EE value among neighbor nodes of the node as its parent node. We have to consider the residual energy of the next hop node because 
transmitting data in high frequency will dissipate a large sum of energy. In this way, the node can guarantee not only the communication but also the effective consumption of energy. In low frequency situation, the reason why we only employ ETX metric is that we firstly guarantee the communication quality, and the node will not consume much energy in this state. Concrete actions the node undertakes are illustrated below.

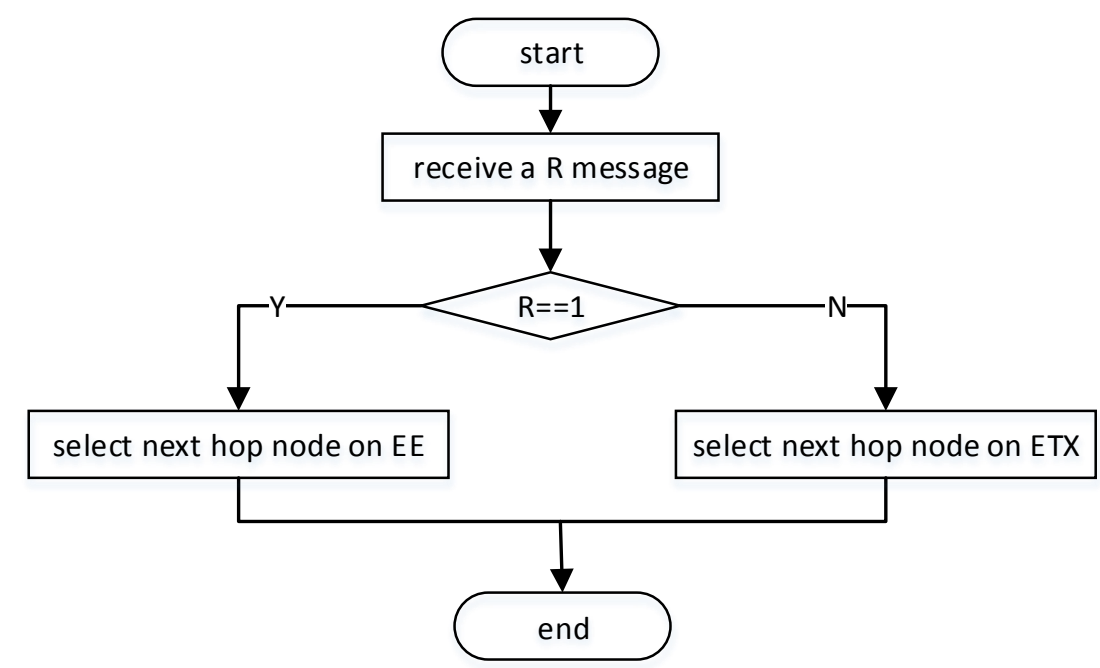

Figure 5. The Flow Chart of Routing Selecting Strategy

When the node monitors the emergency, the path including the node will form a new routing on EE, while other paths that do not include the node's transmission path will keep the routing selecting strategy based on ETX. To achieve this, we utilize a one bit of route change message, called $\mathrm{R}$ embedded in the reserved field in CTP data frame. As shown in Fiigure.5, if the $\mathrm{R}$ value received by a node is equal to 1 , the node will select its next hop node on EE, else on ETX. Firstly the R value is defined as 0 , it will become 1 when the node monitors the emergency. It will reset to 0 when the node backs to original transmission rate. Its parent node receives the $\mathrm{R}$ value do as its descendant nodes.

\section{Results and Evaluation}

To evaluate efficiency and performance, we have implemented CTP, TEEN and TDRHN in the real experiments. We employ transmission frequency, average voltage dissipation and the number of nodes alive in the network as the main reference standards, so we can compare the performance of TDRHN, TEEN and CTP. In addition, we have to observe the phenomenon whether the path including the node has monitored the burst situation change its routing strategy between EE and ETX.

In TinyOS testbed, protocols above have been tested by the TelosB motes, each of which has a CC2420 RF flash chip based on IEEE802.15.4 standard, a small volume RAM supporting limited computing embedded in a low power MCU MSP430. As shown in the Figure.6, we use 10 TelosB modes in our laboratory. The node responsible for receiving other nodes' data of the global network is defined as the sink node, it is connected with the computer with having no power limit. So we do not consider the sink into account in counting energy dissipation aspect and the number of nodes alive. In our experiments the nodes are deployed at fixed locations. 


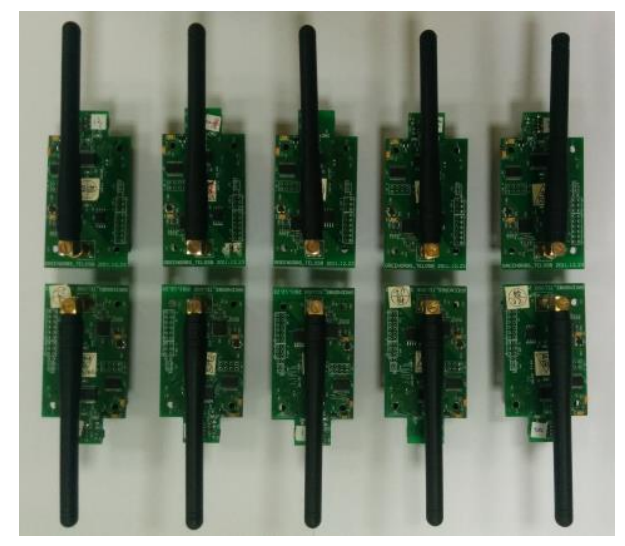

Figure 6. TelosB Motes

We employ the original frequency to transmit a packet per 32 seconds. In our experiments, AT is defined as $45^{\circ} \mathrm{C}$ and $\mathrm{RT}$ is equal to $5^{\circ} \mathrm{C}$. The $\mathrm{EE}$ formula is set that $\alpha=3$ and $\beta=-6$. Here the $\mathrm{N}$ value is set equal to 2 . The transmission actions of different protocols can be deployed by the change of the Node 10's temperature over time.

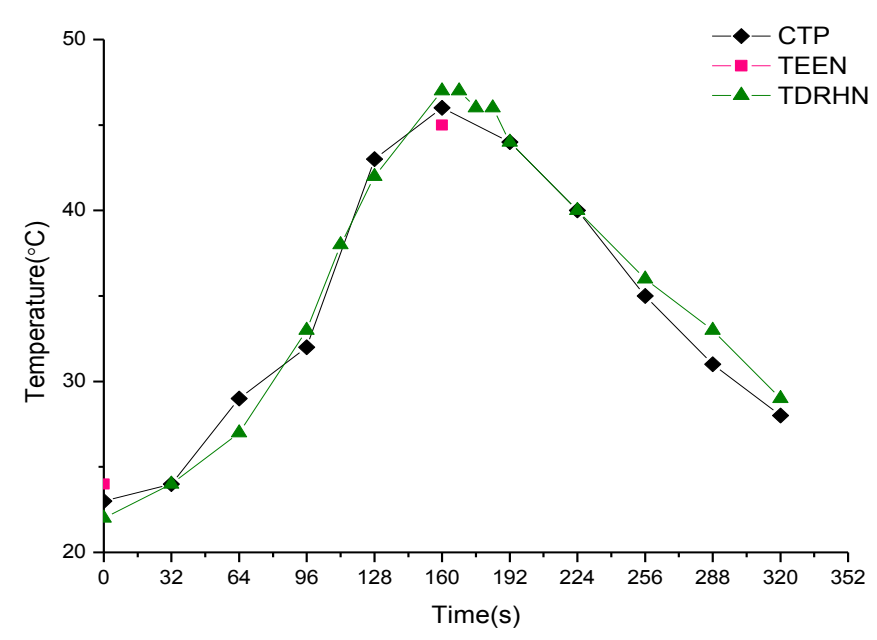

Figure 7. The Temperature of Node 10

Figure.7 displays the temperature of Node 10 over time, we see that TDRHN can dynamically adjust the transmission frequency. The point in the figure indicates one data packet. At first, the node transmits one packet per 32s, when the temperature rises from $27^{\circ} \mathrm{C}$ to $33^{\circ} \mathrm{C}$, at the time $96 \mathrm{~s}$, the condition that converts the original rate to $\mathrm{N}$ times of original rate becomes true, the node sends one packet per 16s. After that, the node's temperature change is less than $5^{\circ} \mathrm{C}$, it backs to the original situation. At the time $160 \mathrm{~s}$, the node's temperature rises up to $47^{\circ} \mathrm{C}$, greater than $45^{\circ} \mathrm{C}$, and the increment value is not less than $5^{\circ} \mathrm{C}$, so the node transmits one packet per $4 \mathrm{~s}$. When the node's temperature is less than $45^{\circ} \mathrm{C}$, at the time $192 \mathrm{~s}$, the node backs to the original transmission state.

In contrast, CTP can not react to the emergency, just periodically sending data. However, TEEN just sends one packet after the first action and does nothing after that. 


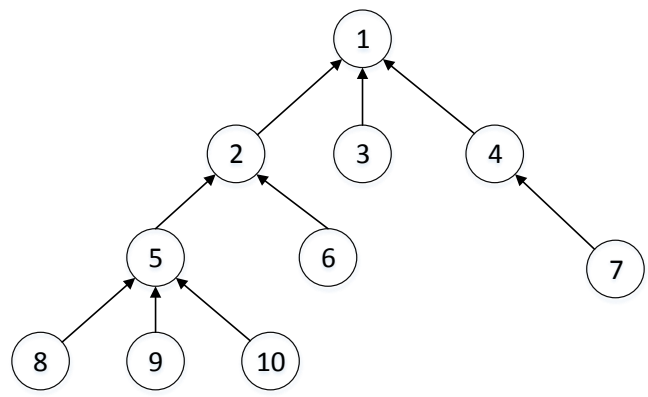

Figure 8. The Topology in Normal Situation

We record the global topology of the network in the experiments. Figure. 8 depicts the topology of the network in normal situation. At this time, to guarantee the communication quality, all the routes form routing path on the basis of ETX. All the nodes take low frequency of sending data to the root.

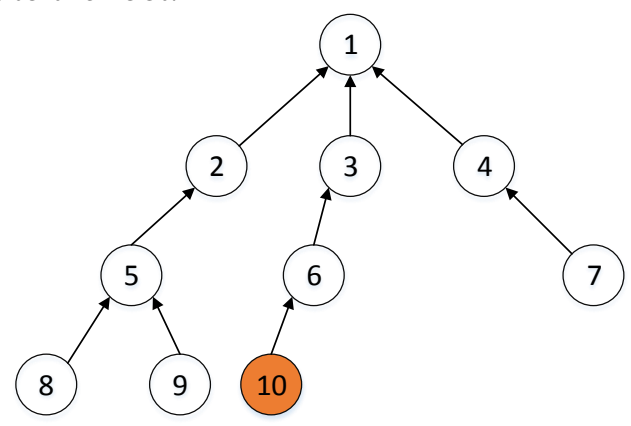

Figure 9. The Topology when Node 10 Monitors the Emergency

When Node 10 detects the emergency, it will immediately employ higher frequency of transmitting data. Simultaneously, it will select Node 6 as its next hop node, Node 6 receives the value of $R$ equal to 1 . Then Node 6 selects Node 3 to transmit its data. By doing above, the path $(10 \rightarrow 6 \rightarrow 3 \rightarrow 1)$ forms routing path on EE while other paths keep the routing on ETX as shown from Figure.9.

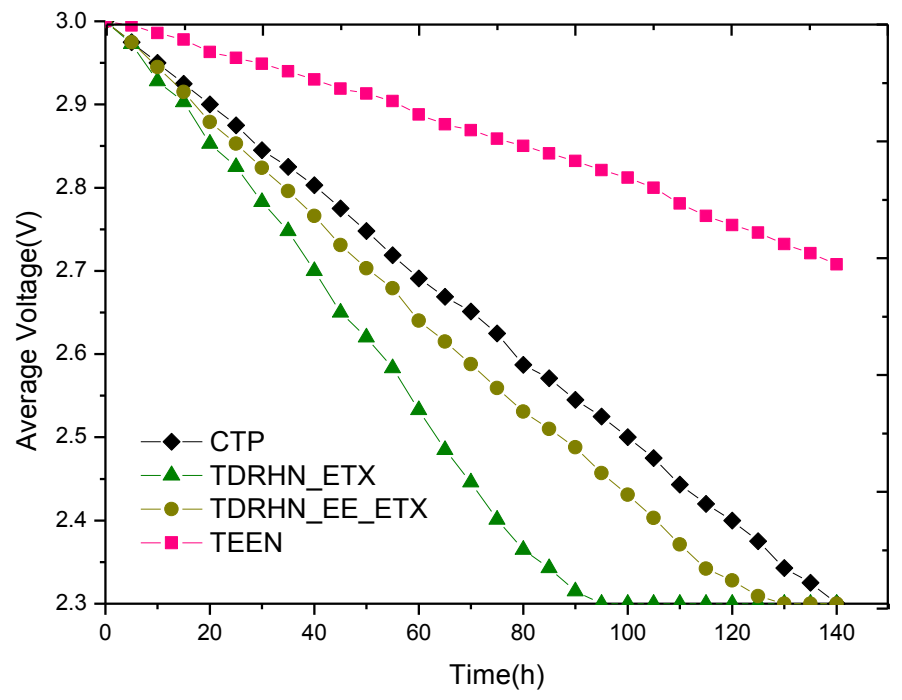

Figure 10. The Average Voltage Change of the Nodes 
Each node equipped with two $1.5 \mathrm{~V}$ AA batteries has $3 \mathrm{~V}$ voltage at the beginning. By analyzing the collected data in our experiments, we find that many errors appear in the data when the voltage is below $2.3 \mathrm{~V}$, therefore we consider $2.3 \mathrm{~V}$ as the death voltage of a node.

To evaluate the energy dissipation performance of TDRHN, we actually employ the voltage value to stand for the energy in the nodes, we can see the energy dissipation situation of the nodes by the voltage change over time. Because the sink is connected with adequate power with having inexhaustible power, so we just compute the average voltage of 9 nodes exception of the sink and death time of them. We has tested two sorts of TDRHN, one is TDRHN_ETX that can dynamically adjust the transmission rate without adjusting the routing selecting strategy, representing the routing metric is fixedly based on ETX; the other is TDRHN_EE_ETX that can dynamically adjust not only the transmission frequency but also the routing selecting strategy, representing the routing metric can switch between ETX and EE. In the experiments, we again set one packet per second as the original transmission rate of TDRHN_ETX, the TDRHN_EE_ETX is the same. CTP as well as TEEN employs the rate to transmit one packet per second. Here the $\mathrm{N}$ value is defined as 4. The emergency is defined as the above. Figure.10 shows the voltage change of each protocol. We observe TEEN descends the least voltage in the same time, because it does not send data without detecting the burst situation that it defines. TDRHN_ETX declines the most voltage comparing with others, because it converts the slow transmission rate to high rate when the node monitors the emergency, and its routing keeps the same as original. Though TDRHN_EE_ETX transmits data in high rate when the node monitors the emergency, the voltage descent trend of TDRHN_EE_ETX approximates that of CTP, that's because it dynamically adjust the routing metric according to the environment situation.

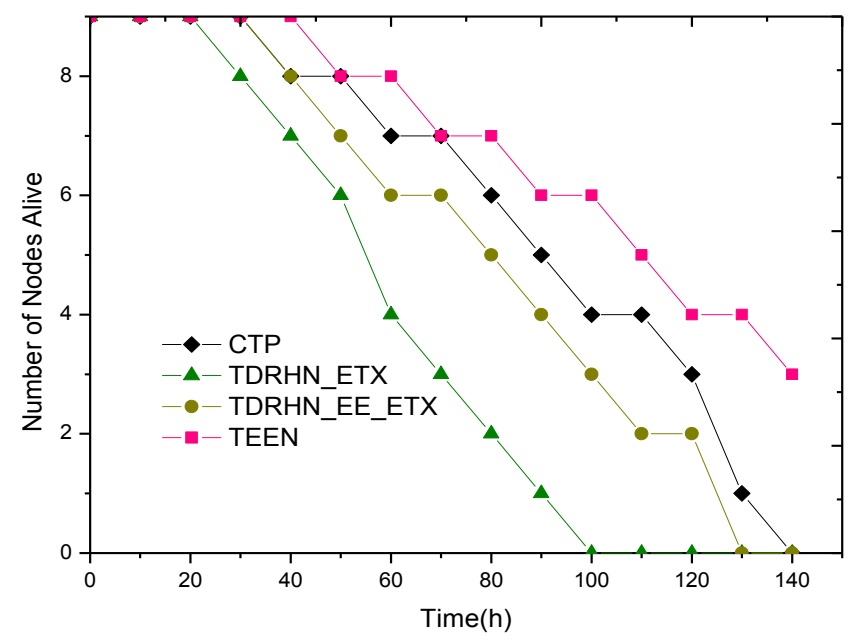

Figure 11. The Number of Nodes Alive

Figure.11 shows the number of nodes alive in the network by testing different protocols. As shown from the figure, we can see TEEN's dead nodes are the least, because TEEN do not send data in normal situation. TDRHN_ETX dissipates the most energy in the same time, and the death speed of the nodes is the fastest. The death speed of TDRHN_EE_ETX's nodes is about the same as that of CTP, demonstrating the outstanding contribution of dynamically adjusting the routing selecting strategy.

From the experiments and results above, we can conclude that TDRHN is an effective protocol for hybrid networks, it can't only collect data in normal situation but also react to the emergency. It saves the energy by dynamically adjusting routing, which will not 
dissipate much energy in the state of fast transmission rate.

\section{Conclusions and Future Work}

This paper proposes TDRHN for hybrid networks, it can not only persistently collect data in normal situation but also support the response to emergencies. As the node employs high frequency of transmitting data when it monitors the emergency, the route including the node immediately forms a new route on EE instead of ETX. When the node returns to normal state, the transmission rate will become to the original state, and the route including the node will form a path based on ETX instead of EE. So TDRHN can be used in many different applications. The high frequency of transmitting data still dissipates energy quickly. In the future, the nodes transmitting a number of data may be considered to find multi-paths to transmit data.

\section{Acknowledgements}

This work is supported by the NSFC (61232016, U1405254, 61173141, 61173142, 6 1173136, 61373133), 201301030, 2013DFG12860, BC2013012 and PAPD fund.

\section{References}

[1] I F Akyildiz, W Su, Y Sankarasubramaniam, E Cayirci. “A survey on sensor networks"[J]. Communications magazine, IEEE, vol. 40, no. 8, (2002), pp. 102-114.

[2] A. Mainwaring, D. Culler, J. Polastre, R. Szewczyk, J. Anderson, "Wireless sensor networks for habitat monitoring" [C]. Proceedings of the 1st ACM international workshop on Wireless sensor networks and applications. ACM, (2002), pp. 88-97.

[3] M Ding, D Chen, K Xing, X Cheng. "Localized fault-tolerant event boundary detection in sensor networks"[C]. INFOCOM 2005. 24th Annual Joint Conference of the IEEE Computer and Communications Societies. Proceedings IEEE. IEEE, no. 2, (2005), pp. 902-913.

[4] O. Gnawali , R. Fonseca , K Jamieson, D Moss, p Levis. "Collection tree protocol"[C]. Proceedings of the 7th ACM Conference on Embedded Networked Sensor Systems. ACM, (2009), pp. 1-14.

[5] A. Manjeshwar, D P. Agrawal, "TEEN: a routing protocol for enhanced efficiency in wireless sensor networks"'[C]. null. IEEE, (2001): 30189a.

[6] W R Heinzelman, A Chandrakasan, H. Balakrishnan H. "Energy-efficient communication protocol for wireless microsensor networks"'[C]. System sciences, 2000. Proceedings of the 33rd annual Hawaii international conference on. IEEE, vol. 2, no. 10, (2000).

[7] W. Xiao, L. Xie, J. Chen, L. Shue. "Multi-step adaptive sensor scheduling for target tracking in wireless sensor networks"[C]. Acoustics, Speech and Signal Processing, 2006. ICASSP 2006 Proceedings. 2006 IEEE International Conference on. IEEE, (2006), 4: IV-IV.

[8] B N Mahajan, V M Thakare, R V Dharaskar, "Dynamic route and threshold level selection in energy efficient WSN"[C]. Emerging Trends in Engineering and Technology (ICETET), 2010 3rd International Conference on. IEEE, (2010), pp. 797-802.

[9] J. Zhang, Z. Yang , B Wang, H Sun , X Sun, "E-CTP: An Energy-balanced Collection Tree Protocol for Power Constrained Wireless Sensor Networks"[J]. International Journal of Grid and Distributed Computing, vol. 7, no. 2, (2014), pp. 115-126.

[10] A. Cerpa, J L Wong, M. Potkonjak, D. Estrin,“Temporal properties of low power wireless links: modeling and implications on multi-hop routing" $[C]$. Proceedings of the 6th ACM international symposium on Mobile ad hoc networking and computing. ACM, (2005), pp. 414-425.

[11] J. Shen, H. Tan, J. Wang, J. Wang, S. Lee, "A Novel Routing Protocol Providing Good Transmission Reliability in Underwater Sensor Networks"[J]. Journal of Internet Technology, vol.16, no. 1, (2015), pp. 171-178.

[12] S. Xie, Y. Y Wang, "Construction of Tree Network with Limited Delivery Latency in Homogeneous Wireless Sensor Networks"[J]. Wireless personal communications, vol. 78, no. (1), (2014), pp. 231-246. 


\section{Authors}

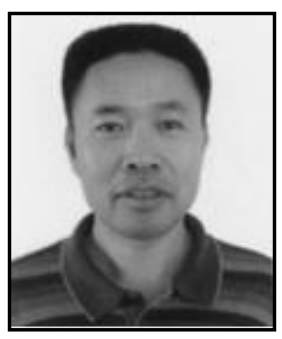

Xingming Sun, He is a professor in the School of Computer and Software, Nanjing University of Information Science and Technology, China from 2011. He received the B.S. degree in Mathematical Science from Hunan Normal University and M.S. degree in Mathematical Science from Dalian University of Technology in 1984 and 1988, respectively. Then, he received the $\mathrm{Ph} . \mathrm{D}$. degree in Computer Engineering from Fudan University in 2001. His research interests include information security, network security, cryptography and ubiquitous computing security.

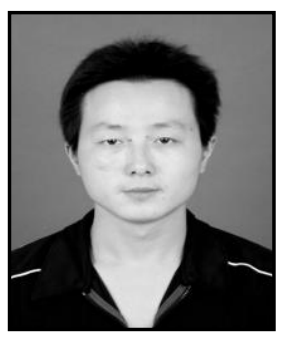

Chengju Xue, He received his B.S. degree in Software Engineering from Nanjing University of Information Science and Technology, China in 2013. Currently he is studying for his M.S. degree in Software Engineering at the same university. His research interests include wireless networks and network routing.

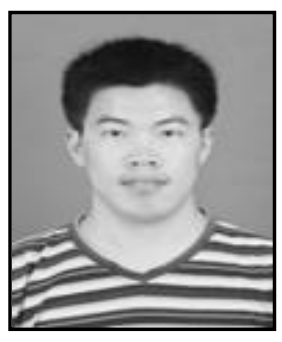

Baowei Wang, He received his B.S. and Ph.D. degrees in Computer Science from Hunan University in 2005 and 2011, respectively. He is currently working as a lecturer in School of Computer and Software, Nanjing University of Information Science and Technology. His research interests include steganography, wireless networks and securing ad hoc networks. 